\title{
Microbubble Axial Localization Errors in Ultrasound Super-Resolution Imaging
}

\author{
Kirsten Christensen-Jeffries, Sevan Harput, Jemma Brown, Peter N. T. Wells, Paul Aljabar, Chris \\ Dunsby, Meng-Xing Tang, and Robert J. Eckersley.
}

\begin{abstract}
Acoustic super-resolution imaging has allowed visualization of microvascular structure and flow beyond the diffraction limit using standard clinical ultrasound systems through the localization of many spatially isolated microbubble signals. The determination of each microbubble position is typically performed by calculating the centroid, finding a local maximum, or finding the peak of a 2-D Gaussian function fit to the signal. However, the backscattered signal from a microbubble depends not only on diffraction characteristics of the waveform, but also on the microbubble behavior in the acoustic field. Here, we propose a new axial localization method by identifying the onset of the backscattered signal. We compare the accuracy of localization methods using in vitro experiments performed at 7 cm depth and $2.3 \mathrm{MHz}$ center frequency. We corroborate these findings with simulated results based on the Marmottant model. We show experimentally and in simulations that detecting the onset of the returning signal provides considerably increased accuracy for super-resolution. Resulting experimental crosssectional profiles in super-resolution images demonstrate at least 5.8 times improvement in contrast ratio and more than 1.8 reduction in spatial spread (provided by $90 \%$ of the localizations) for the onset method over centroiding, peak detection and 2D Gaussian fitting methods. Simulations estimate that these latter methods could create errors in relative bubble positions as high as $900 \mu \mathrm{m}$ at these experimental settings, while the onset method reduced the interquartile range of these errors by a factor of over 2.2. Detecting the signal onset is therefore expected to considerably improve the accuracy of super-resolution.
\end{abstract}

This research was supported by the Department of Health via the National Institute for Health Research (NIHR) comprehensive Biomedical Research Center award to Guy's and St Thomas' NHS Foundation Trust in partnership with King's College London and King's College Hospital NHS Foundation Trust. The Centre of Excellence in Medical Engineering funded by the Wellcome Trust and EPSRC under grant number WT 088641/Z/09/Z. Additional funding provided by the EPSRC to King's College London and Imperial College London under grant numbers EP/N14855/1 and EP/N015487/1 respectively. The views expressed are those of the author(s) and not necessarily those of the NHS, the NIHR or the Department of Health.

K. Christensen-Jeffries, J. Brown, P. Aljabar and R. J. Eckersley are with the Biomedical Engineering Department, Division of Imaging Sciences, Kings College London, London, UK (phone: 0207188837; e-mail: kirsten.christensen-jeffries@kcl.ac.uk and robert.eckersley@kcl.ac.uk).

C. Dunsby is with the Department of Physics Imperial College London, London, SW7 2AZ, UK and the Centre for Pathology, Imperial College London, London, W12 0NN, UK (email: christopher.dunsby@imperial.ac.uk).

S. Harput and M. X. Tang are with the Department of Bioengineering, Imperial College London, London, SW7 2AZ, UK (email: s.harput@imperial.ac.uk and mengxing.tang@imperial.ac.uk).

P. N. T. Wells is with the School of Engineering at Cardiff University, Cardiff, Wales, CF24 3AA, UK.
Index Terms - Biomedical imaging, Microbubbles, Microvasculature, Ultrasonic imaging, Ultrasound, Resolution.

\section{INTRODUCTION}

$T$ here is a clinical need to non-invasively detect architectural changes and flow variations in the microvasculature for the early detection and intervention of diseases such as cancer [1], [2] and peripheral arterial disease [3]-[5].

Currently, visualization of the vasculature in clinical ultrasound (US) can be achieved using microbubble contrast agents [6]-[8]. These gas filled spheres, between 1-7 $\mu \mathrm{m}$ in diameter, have a resonance frequency which falls within the 1 to $15 \mathrm{MHz}$ range conventionally used in diagnostic US imaging [9]. Thus, microbubbles return considerably stronger echoes than similar sized tissue scatterers such as ensembles of red blood cells. This, along with the additional advantage that their size means they remain within the vasculature, makes them an ideal vascular contrast agent.

However, US faces a limit inherent to all wavebased imaging processes, where diffraction of the transmitted and received waves causes point sources to become indistinguishable from one another when they reach a proximity which is approximately half the wavelength of the transmitted wave. Beyond this, interference of the sound scattered from these sources results in acoustic speckle. The diffractionlimited image resolution fundamental to the conventional clinical US frequency range, approximately $50-750 \mu \mathrm{m}$, is unable to adequately resolve the microvasculature. The use of shorter wavelength pulses by transmitting high frequency US has been implemented as a means of obtaining high-resolution imaging [10], however 
these techniques are limited to shallow depths due to the inherent compromise between higher frequencies and attenuation.

Sub-diffraction imaging has been a revolutionary technology in optical microscopy [11]-[16], where methods such as photo-activatable localization microscopy (PALM) [15] and stochastic reconstruction microscopy (STORM) [16] have been developed to overcome the same fundamental physics through the localization of many spatially isolated photo-activatable fluorophore signals. In these approaches, fluorescent particles within a diffraction limited region are activated at different time points so that they can be individually imaged, localized, and subsequently deactivated. Here, the position determination is usually done by fitting a 2D Gaussian ( $x-y$ vs. photon number) to the emission intensity distribution of the fluorescent molecule [17],[18]. An image of the biological structure is then generated using the positions of the fluorophores labelling the structure. These techniques have enabled visualization of nanoscale structures far smaller than the conventional fluorescence microscopy resolution limit $(\sim 250 \mathrm{~nm}$ laterally and $600 \mathrm{~nm}$ axially) [19].

Since microbubbles are considerably smaller than the US resolution limit, spatially isolated individual bubbles can be considered as point scatterers in the acoustic field. This, along with their function as a vascular marker, allows analogous US techniques to be developed to overcome the conventional diffraction limit. The localization of many spatially isolated microbubble signals has recently been applied in US with the aim of replicating the gains seen in optical microscopy.

US super-resolution (US-SR) images that demonstrate the resolution of two sub-diffraction features in vitro were shown in our previous work [20], using clinical imaging frequencies and a low microbubble concentration ensuring a high probability of spatially isolated signals,. We have since been able to provide maps of the fine microvasculature in vivo in an unclamped mouse ear using around 12000 frames (under 9 minutes of US acquisition) [21]. This work used an unmodified clinical US system in a standard contrast enhanced mode and therefore requires only equipment already present in the clinic. Siepmann et al, 2011 [22] demonstrated a similar localization-based method to increase the resolution of vascular images; however, the resolution of the resulting features was not evaluated. This was performed at a high frequency of $40 \mathrm{MHz}$; at this frequency, a wavelength $\sim 40 \mu \mathrm{m}$ is already able to provide high resolution; in addition, penetration depth is limited by the use of high imaging frequencies. This group and others have also successfully demonstrated in vivo results using various localization-based methods in a stationary rat brain at high clinical frequency (15 $\mathrm{MHz}$ ) using high frame rates (HFR) [23], in tumorbearing mice at $30 \mathrm{MHz}$ [24] and at $4.5 \mathrm{MHz}$ with HFR [25].

Super-resolution localization requires the identification of some feature from the returned signal that is thought to approximate the bubble's position. To the best of our knowledge, in all previous publications regarding US superresolution, the microbubble position is assumed to coincide with the maximum amplitude, center of mass, or a related measure, of the backscatter signal. Using these techniques, each microbubble from the insonated bubble population is expected to behave in the same way, hence generating a signal close to the system PSF and as such the determination of each microbubble position is typically done by calculating the centroid [20]-[22], finding a local axial maximum from the travelling hyperboloid in RF data lines and fitting a function, such as a parabola in the case of Desailly (2013) [26], cross-correlation of signals with an expected response [24], or fitting a 2-D Gaussian function either to the original beamformed backscatter signal [27], or after deconvolving with a predicted Gaussian PSF [23].

In the axial direction, the chosen feature is likely to introduce a bias to the true position of the bubble; this bias depends upon which feature of the transmit pulse is defined as the initial time, $t_{0}$ within the US system. In clinical systems, this is likely to be unknown. Nevertheless, a consistent bias which occurs for every measurement does not cause a significant problem for US-SR imaging, since the 
combination of many localizations with constant offset will still generate an accurate US-SR image, while its real depth may be a few $100 \mu$ m shallower or deeper. However, variations in this position offset between different detected bubble signals can cause substantial inaccuracies that vary in magnitude. This can have significant consequences for all localization-based US-SR techniques through the axial blurring of super-resolution images.

It is important to note that the work presented in this paper does not aim to investigate the fundamental limit to which a single, linear, point scatterer can be localised with a given localisation method, as has been investigated in other work [28]. The work presented here differs for a number of reasons. Firstly, our study involves the comparison of a number of different localisation methods. Our aim was to establish which method of localisation is least affected by signal variations and hence most accurate at determining the relative position of the bubbles, crucial for super-resolution imaging. Furthermore, the fundamental resolution limit was investigated previously only in the lateral direction experimentally, while here we are concerned with axial localisation. Secondly, the previous literature does not account for the huge variation in bubble responses, and as such backscatter signals are analysed assuming they can be approximated as that from an ideal linear point scatterer. This variation affects each localisation algorithm differently. Within our manuscript, it is the huge variability in microbubble signal responses due to properties of the microbubbles themselves, and of their environment, that is the problem that we aim to solve for future super-resolution imaging.

The scattered signal received from each single microbubble depends not only on diffraction characteristics of US transmission and reception, but also on the bubble's behavior in the acoustic field. The emitted sound pressure from a compressible body capable of volume oscillations insonated by an incident wave is composed almost entirely from the active emission of sound caused by these oscillations [29], rather than the passive contribution due to the perturbation of the density field in surrounding fluid caused by the presence of the object in the path of the sound wave.

The strength and duration of microbubble volumetric oscillations is likely to depend upon properties of the transmit pulse, such as the number of cycles, transmit pressure and frequency, as well as properties of individual bubbles including the bubble's size [30], [31], [32], its location in the field of view [33], its proximity to neighboring objects or bubbles [34],[35], and its shell composition [36], [37]. However, while the spatially variant changes in PSF can be predicted and addressed, the unpredictable, stochastic behaviour of a polydispersed population of bubbles, each with a unique shell composition, makes it challenging to predict both their linear and non-linear signal response. The number of cycles, pressure, and frequency of the transmit pulse are likely to have a predictable influence on the microbubble linear response, whereas the non-linear response is not straightforward to model. Nevertheless, these factors are typically invariant during the course of acquisition, and thus will not influence the spread of errors during super-resolution image generation.

With a fixed transmit frequency, the polydispersed microbubble distribution, characteristic of most contrast agent solutions, will give rise to responses ranging from fully resonant, to non-resonant bubbles [38]. Resonant bubbles will oscillate strongly, giving a large and sometimes elongated backscatter intensity due to continuing oscillations, while bubbles larger or smaller than this will give a significantly reduced signal. In addition, the insonating pressure experienced by each bubble will vary depending on its position within the acoustic field. Its proximity to objects, vessel boundaries, and neighboring bubbles can damp or alter its response to the transmission pulse [34], [35], [39], [40].

The lateral localization position, however, is unlikely to be affected by the duration and asymmetry of the time profile, and instead a position indicative of the lateral center of the returned signal should represent the position of the underlying scatter source. Each of these factors will, however, contribute to wide axial variations in returning microbubble signals which causes 
problems for localization methods which rely upon predictable or ideal scatter behavior.

Centroiding allows for position estimation using intensity weighting, and therefore this method is strongly affected by signal tails and asymmetry in the axial scatter response. Similarly, 2D Gaussian fitting methods (with variable or fixed width) will be influenced by asymmetrical or elongated intensity distributions. These methods and others like it that define an 'expected response', assume a pre-determined signal shape and/or size; thus, deviations from this will result in inaccurate localizations. Furthermore, to limit this effect, such methods often reject signals which differ considerably from the defined expectation [24][27], which reduces the localisation rate and thus increases the acquisition time required to generate super-resolution images. Localization methods based on peak finding are susceptible to irregular or distorted signals, in addition to noise fluctuations.

Quantifying a microbubble signal using the first point at which the intensity distribution has increased to $50 \%$ of its maximum would remove effects from ringing signals occurring after the peak. This, however, still relies upon the magnitude of the scattered response to define the localization position. Variation in the signal peak amplitude will therefore cause a shift in the axial localization position that depends upon the gradient of the signal onset.

The leading edge of the returned signal should instead be indicative of the true position of the bubble. A new localization algorithm is therefore proposed to estimate the onset of the backscatter signal, defined using a noise threshold, which should be unaffected by elongated, tailing or distorted signals, and therefore should more accurately determine the relative position of the insonated objects.

Here, we present a study of the accuracy of these six localization methods used to identify the position of each microbubble from its scattered signal. The accuracy of these methods is evaluated using simulated responses from a polydispersed bubble population to a measured experimental transmit wave, and findings are corroborated in vitro using a capillary tube flow phantom, designed to assess imaging performance at depths of up to 7 $\mathrm{cm}$. Qualitative and quantitative analysis of the data is performed in order to better understand the origin of the underlying signals. We show that localization methods which identify the onset of the returning microbubble signal are required to obtain good positional accuracy in super-resolution imaging.

\section{Materials And Methods}

\section{A. Simulation}

The localization accuracies of the methods were tested with a simulation model using pulse inversion processing. The pressure output of the clinical US scanner was measured and used as an input to the simulations. This was determined using a $0.5 \mathrm{~mm}$ needle hydrophone (SN 1832, Precision Acoustics Ltd., Dorchester, UK). The center frequency of the measured signal was $2.3 \mathrm{MHz}$ and the $-6 \mathrm{~dB}$ bandwidth was $1.7 \mathrm{MHz}$. The excitation waveform was inverted to generate a negative pulse that will achieve complete cancellation during pulse inversion (PI). Each pulse inversion pair had a peak-negative-pressure (PNP) of $46 \mathrm{kPa}$ for the positive pulse and $64 \mathrm{kPa}$ for the negative pulse due to the non-symmetric nature of the Gaussian pulse.

Simulations included white noise with SNR value of $20 \mathrm{~dB}$. This SNR was calculated using a reference contrast-enhanced frame from the experimental data acquired by the US scanner and the simulated signal from a microbubble with $1 \mu \mathrm{m}$ radius. Noise was added to the pressure waveform before filtering with a linear phase finite impulse response bandpass filter.

Marmottant's model was implemented to simulate the behavior of Sonovue microbubbles with parameters chosen according to $\mathrm{Tu}$ et al, 2009 [41] and Marmottant et al, 2005 [42]. Simulations were repeated 31,400 times using a weighted distribution of microbubbles with 2-9 $\mu \mathrm{m}$ diameter range to cover the behavior of a native Sonovue population according to Gorce et al, 2000 [31]. The radial microbubble oscillations simulated by this model were converted into a pressure waveform at $7 \mathrm{~cm}$ depth. Bubble localisations were then performed as described in Section B5). 


\section{B. Experiment}

\section{1) Ultrasound Equipment and Acquisition} Imaging was performed using a standard, unmodified Siemens Acuson Sequoia clinical US scanning system (Siemens, Issaquah, WA) with a $4 \mathrm{C} 1$ abdominal convex probe with focal zone of $5-7 \mathrm{~cm}$. Imaging settings were as follows: dynamic range $100 \mathrm{~dB}$; TGC $-20 \mathrm{~dB} / \mathrm{cm}$; transmit frequency $2.3 \mathrm{MHz}$; frame rate $25 \mathrm{fps}$ and MI 0.07.

The estimated diffraction limited resolution of the scanner under the settings used here was $239 \mu \mathrm{m}$ and $336 \mu \mathrm{m}$ in the axial and lateral directions respectively, measured as the average FWHM over 50 frames of a stationary point scatterer signal represented by the cross-section of a fine wire.

\section{2) Phantom Set-Up}

Super-resolution imaging was demonstrated using two thin-walled cellulose capillary tubes (Hemophan ${ }^{\circledR}$, Membrana) of internal diameter 200 $\mu \mathrm{m} \pm 15 \mu \mathrm{m}$, a wall thickness of $8 \pm 1 \mu \mathrm{m}$ in the dry state and a length change under wet conditions of \pm $1 \%$ (specifications provided by the manufacturer). The ends of each tube were threaded into butterfly needles and sealed with commercially-available epoxy-resin before being placed in a gasequilibrated water bath. The two tubes were positioned diagonally with their centers touching, such that a crossed-tube structure was within the imaging plane of the transducer; this set-up is illustrated in the schematic shown in Figure 1.

One end of each tubing was placed in a beaker of dilute microbubble solution, and the other was attached to one of two identical syringes. The flow rate of both tubes was controlled using the same syringe pump driver. The opposing tube endings were placed in the same beaker containing a dilute microbubble suspension. Therefore, the flow rate and concentration in both tubes were assumed to be approximately equal. In vitro targets were at a depth of approximately $7 \mathrm{~cm}$ and were positioned on an optical bench (Nexus $^{\mathrm{TM}}$, Thorlabs) to reduce vibrations due to external factors.

\section{3) Ultrasound Imaging Procedure}

A dilute suspension containing $100 \mu \mathrm{l}$ of
Sonovue $^{\mathrm{TM}}$ (Bracco) microbubbles in $600 \mathrm{ml}$ water was drawn through the tubes using the syringe pump at a rate of $100 \mu \mathrm{l}$ per minute. The microbubble suspension was stirred to ensure a uniform distribution of microbubbles within the suspension. Cadence ${ }^{\mathrm{TM}}$ CPS imaging mode was used to acquire 9344 frames, providing an average of approximately 5 signals per frame.

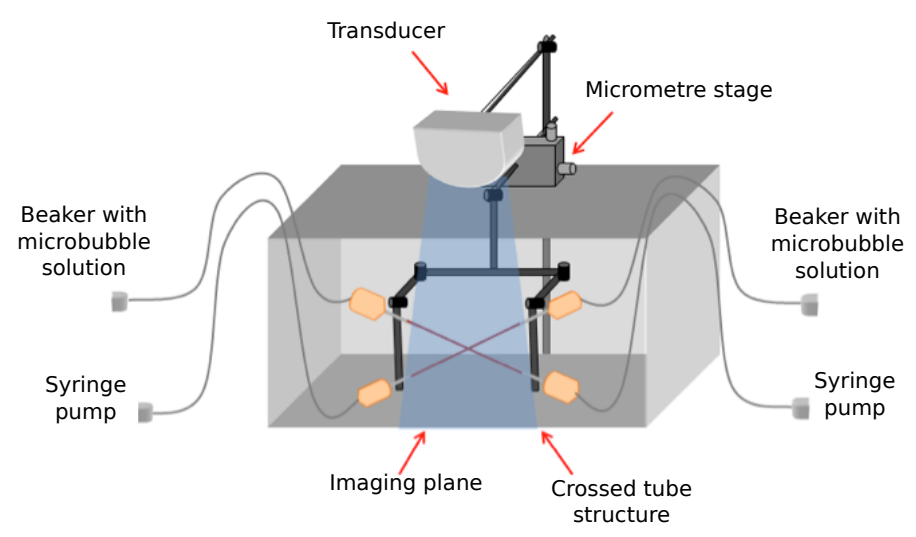

Figure 1. US imaging set-up for crossed tube experiments. Both tubes were connected to separate syringes within the same syringe pump driver; hence the flow rate was kept approximately equal in both systems.

\section{4) Microbubble Identification}

Characterisation of the noise in the system was performed on 50 control frames. These were acquired before the introduction of microbubbles and were assumed to be representative of background noise. The background intensity level for each frame was defined as the $99^{\text {th }}$ percentile of the pixel intensities and is employed here to ensure the threshold is above the large majority of intensities represented by noise, but is not affected by any outlier pixels within the background frames. The mean amplitude of all background intensity levels determined for each frame was used as an intensity threshold to eliminate background noise in the data frames.

Potential bubble frames were then segmented into foreground and background according to whether the pixel values were above this background intensity level. Characteristics of each connected region in the foreground were then calculated for analysis. Empirically determined lower and upper size thresholds were used to reject noise $(<0.01$ $\mathrm{mm}^{2}$ ) and multiple bubble signals which were not 
spatially separated $\left(>1.7 \mathrm{~mm}^{2}\right)$. Remaining regions were classified as bubbles and localization methods described in the following section were performed.

\section{5) Microbubble Localization}

Six localization methods described below (a-f) were tested and are illustrated in Figure 2. For methods a$\mathrm{d}$, the lateral intensity weighted center of mass provided the microbubble localization x-coordinate, $C_{x}$. Microbubble localization in the axial direction, coordinate $C_{y}$, was performed on the axial bubble profile. This profile was the averaged axial signal from pixel columns at and either side of the $\mathrm{x}$ localization position after cubic interpolation. For methods $e$ and $f, 2 D$ Gaussian fitting techniques were performed on the 2D image data from detected signals to determine both $C_{x}$ and $C_{y}$.

\section{a) Method 1: Centroiding}

The axial centroid was found by calculating the intensity weighted center of mass of each extracted bubble signal.

\section{b) Method 2: Onset}

Detection of the beginning of the bubble signal was performed by locating the point at which the axial profile exceeded three standard deviations above the noise level $(99.73 \%$ of the noise assuming a normal distribution).

c) Method 3: 50\% Point

Method 3 involved calculating the position at the leading edge of the axial bubble signal profile where the signal reached $50 \%$ of its maximum value.

\section{d) Method 4: Peak}

Method 4 detected the peak of the signal along the averaged axial profile.

e) Method 5: 2D Variable-width Gaussian Fit

In this approach, a nonlinear least squares fit to each microbubble signal with a 2D Gaussian function (1D for simulations) was performed, where the amplitude and standard deviations in $x$ and $y$ were allowed to vary. The localization position is the center of the fitted distribution.

\section{f) Method 6: 2D Fixed-width Gaussian Fit}

A nonlinear least squares fit with a 2D Gaussian function (1D for simulations) was additionally performed where the standard deviations of the distribution were fixed at the FWHM of the estimated diffraction limited resolution of the system.

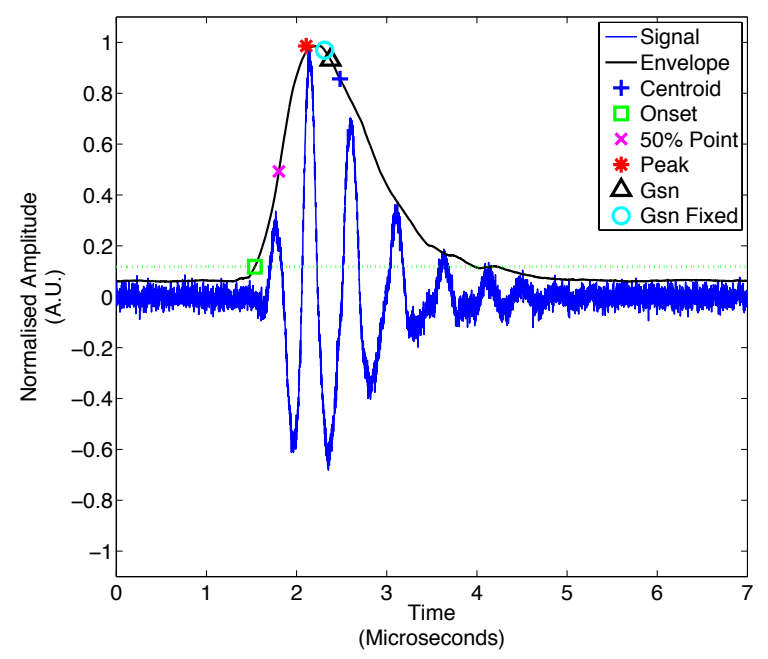

Figure 2. Microbubble localization in the axial direction was performed by estimating the centroid, onset (threshold shown by green dashed horizontal line), $50 \%$ point, peak, and center of a variable-width and fixed-width Gaussian fits. Here, resulting localizations are demonstrated on the simulated scattered pressure from a microbubble with $2 \mu \mathrm{m}$ initial radius. It is important to note that the relative locations vary with each individual microbubble signal.

\section{6) Localization Maps}

Localizations from all acquired frames are then superimposed onto a single map to form an image of the underlying structure containing the microbubbles. Here, each individual bubble localization was represented by a 2-D Gaussian profile centered at $C_{x}, C_{y}$ with standard deviations, $\sigma_{x}$ and $\sigma_{y}$, given by the average lateral and axial localization uncertainty of the PSF FWHM [21]. The super-resolution map resulting from the sum of all the individual centroid localization distributions across all frames creates an image in which the displayed value is proportional to the chance of finding a microbubble positioned at that location.

\section{RESULTS}

\section{A. Simulation}

The pressure output of the clinical scanner measured with a needle hydrophone and 
subsequently used in simulations is shown in Figure $3 \mathrm{~A}$. SonoVue microbubbles with a radius, $\mathrm{R}_{0}$, of 1.6 - $3.2 \mu \mathrm{m}$ are expected to be resonant for this measured acoustic waveform [43]. Figure 3B-D illustrates three characteristic waveforms observed during simulation, demonstrating large variations in amplitude and duration of microbubble responses. Figure 3B shows linear scattering from an offresonance microbubble at low pressure. Figure $3 \mathrm{C}$ and $\mathrm{D}$ display non-linear scattering from a resonant and an off-resonance microbubble (with a large diameter) respectively. The duration of these signals above the noise level exceeds that of the excitation pulse (Figure 3A) and the linear response (Figure 3B). In both $\mathrm{C}$ and $\mathrm{D}$, continued microbubble oscillations cause elongated waveforms with long tails.

Box plots shown in Figure 4 display the distribution of localization errors for each method over 31,400 repetitions. The interquartile range (IQR) of localization errors represents a measure of the blurring effect due to the inaccuracy of detected relative bubble positions. This value, rather than the mean error, represents the potential degradation in US-SR images due to variations in bubble signals. The onset method demonstrated the lowest IQR of $94 \mu \mathrm{m}$, and therefore provided the most accurate detection of relative bubble positions of all tested methods. Considerably larger spreads of localization errors were found using centroiding and variable-width Gaussian fitting $(300 \mu \mathrm{m}$ and 310 $\mu \mathrm{m}$ respectively). Fixed-width Gaussian fitting provided an improved spread compared to that of variable-width fitting $(210 \mu \mathrm{m}$ cf. $310 \mu \mathrm{m})$. Peak detection showed the largest spread of errors of 445 $\mu \mathrm{m}$, while $50 \%$ point shows a slightly smaller spread of localizations $(375 \mu \mathrm{m})$ over the microbubble population.

\section{B. Experiment}

Examples of the variation in single bubble signals observed in data frames are displayed in Figure 5A and $\mathrm{B}$; here, a range in axial extent above the noise floor from $245 \mu \mathrm{m}$ to $1220 \mu \mathrm{m}$ was observed.

Figure $6 \mathrm{~A}$ and $\mathrm{B}$ display images of two microbubble signals found within a single frame, along with their axial profiles shown in Figure 6C
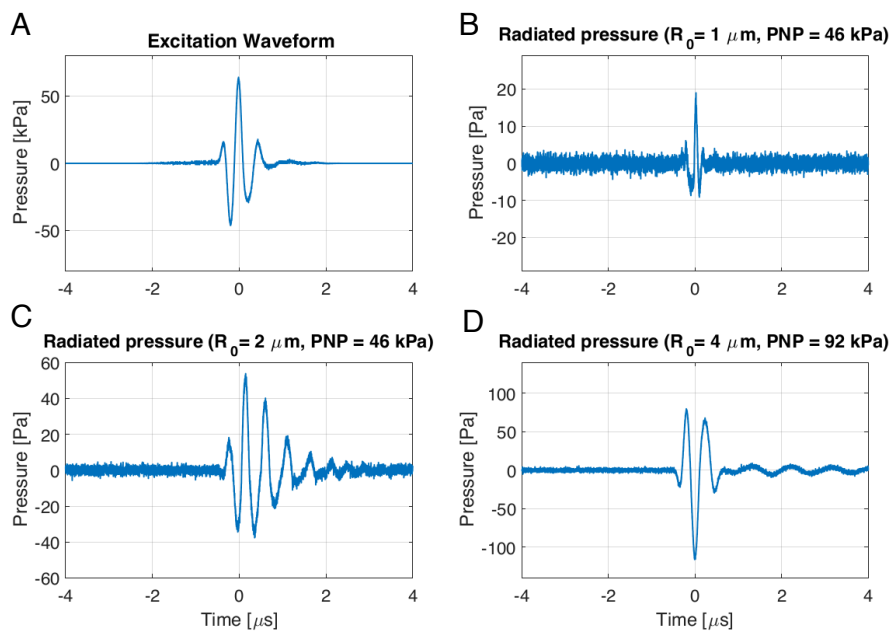

Figure 3A) Pressure output of the US scanner measured with a hydrophone. This waveform was used as an excitation signal in simulations. B) Scattered pressure from a $1 \mu \mathrm{m}$ microbubble at $46 \mathrm{kPa}$. C) Scattered pressure from a 2 $\mu \mathrm{m}$ microbubble at $46 \mathrm{kPa}$. D) Scattered pressure from a $4 \mu \mathrm{m}$ microbubble at $92 \mathrm{kPa}$. Note the difference in y-axis scale for each plot.

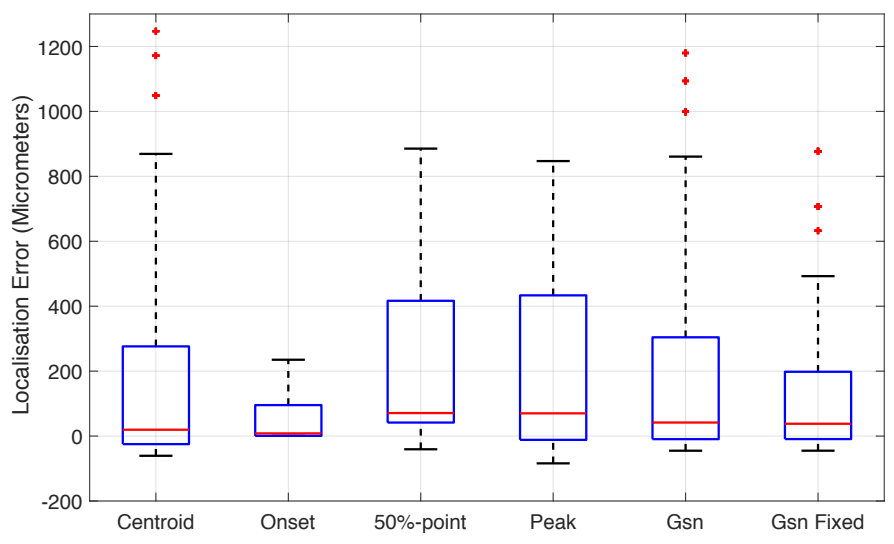

Figure 4. Simulated localization errors from true position using the six tested methods. Error values were calculated for pulse inversion using the excitation waveform measured with a hydrophone (Fig. 9A) over 31,400 repetitions using the SonoVue bubble radius distribution given by Gorce et al. [31]. Whiskers cover approximately $\pm 2.7 \sigma$ and $99.3 \%$ assuming the data are normally distributed.
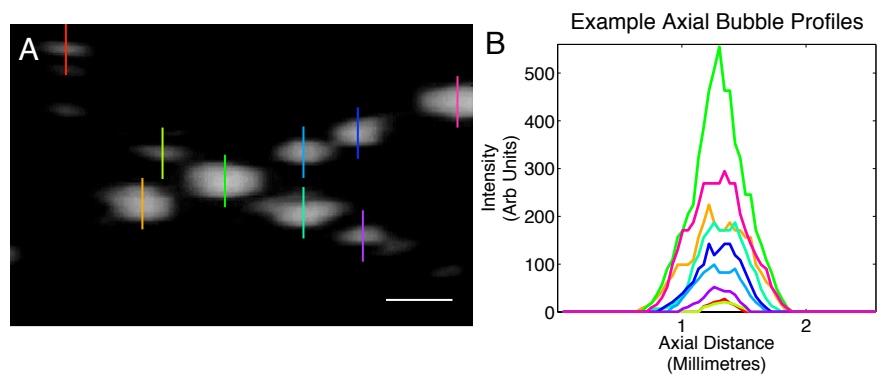

Figure 5.A) Example of spatially isolated bubble signals in crossed tube phantom. Scale bar, $2 \mathrm{~mm}$. B) Profiles show a large variation in both shape and signal amplitude. 
and D. Localizations estimated using each of the six methods are overlaid on all plots. For both bubbles, a large disparity can be observed between the localization positions determined using the different methods. For example, the onset and peak positions are separated by $744 \mu \mathrm{m}$ and $275 \mu \mathrm{m}$ for the bubbles shown in A and B respectively, due to differences in size and shape of the bubbles' axial profiles.

Resulting maps created from localizations detected over all data frames are shown in Figure 7 using each localization method. Here, there is a noticeable blurring of the tube's lower boundary using centroiding (Figure 7A). Both the onset and the $50 \%$ point method provide improved delineation of both upper and lower tube boundaries (Figure 7B and C). Peak detection, shown in Figure 7D additionally demonstrates blurring of the lower tube boundary, and furthermore an additional smoothing filter would be required to reduce the sensitivity of this approach to noise. Flexible Gaussian fitting results in blurring at both the upper and lower boundaries, while fixed Gaussian fitting produces blurring predominantly at the lower edge.

Axial localization profiles summed over four 600 $\mu \mathrm{m}$ sections across the localization maps (red rectangles in Figure 8A) for each of the localization methods are displayed in Figure 8B. There is noticeable spreading of localizations in the profiles using centroiding, peak detection, and both Gaussian fitting methods, and a comparatively lower peak relative to the onset and $50 \%$ point methods. A small tail at depths below the tube is also apparent using the $50 \%$ point method; this method provides the highest peak and demonstrates a generally compact localization distribution. The spatial extent of the onset method demonstrates very little spread beyond the tube diameter.

The mean FWHM of each of the summed profiles, displayed in Figure 9A, is lowest using the $50 \%$ point $(183.5 \mu \mathrm{m})$, followed by the onset method $(201 \mu \mathrm{m})$, while peak detection, centroiding and Gaussian fitting methods all have FWHM values larger than $215 \mu \mathrm{m}$. To estimate the compactness of the majority of the localization distributions, the width of the profile containing
$90 \%$ of the localizations was calculated. This is considerably larger than the nominal tube diameter for centroiding, peak detection, and Gaussian fitting techniques (over $556 \mu \mathrm{m}$ ), demonstrating a large proportion of inaccurate localizations, while the onset and $50 \%$ points are $308 \mu \mathrm{m}$ and $333 \mu \mathrm{m}$, respectively. Furthermore, the interquartile range (IQR) of the localizations is considerably lower for the $50 \%$ point $(59 \pm 29)$ and onset $(64 \pm 30)$ compared to the remaining methods tested. The onset method demonstrates the highest contrast ratio (CR) of $35 \pm 18$, calculated as the ratio of mean signal over $200 \mu \mathrm{m}$ width centered on the peak, to the background over the same width, starting 200 $\mu \mathrm{m}$ from the peak shown in Figure 9D (inset). This is followed by the $50 \%$ point method with a lower CR of $20 \pm 9$, while all the remaining methods do not exceed a CR of 6 .

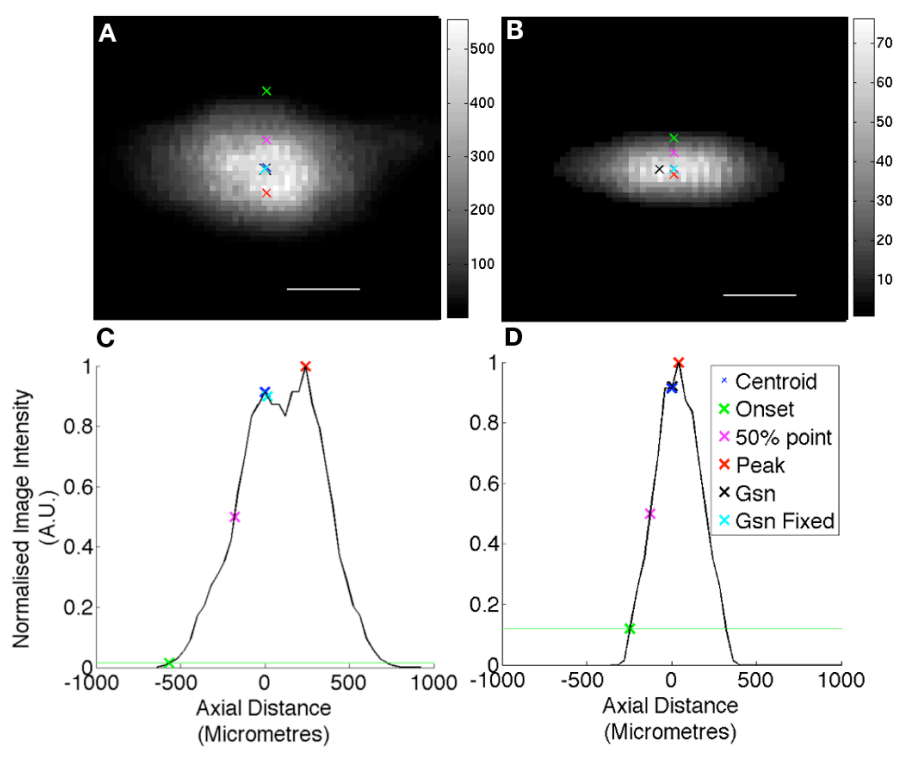

Figure 6. Bubble signals with corresponding localizations by calculating the centroid, signal onset, 50\% point, peak, variable Gaussian fit result, and fixed Gaussian fit result.

An overlay of the localization map created using the onset of each received signal on a diffraction limited image frame with a high microbubble concentration is shown in Figure 10, demonstrating the improved visualization of the fine tubes using this technique. Here, the position of the diffraction limited image of the tubes is noticeably displaced with respect to the localization map. 


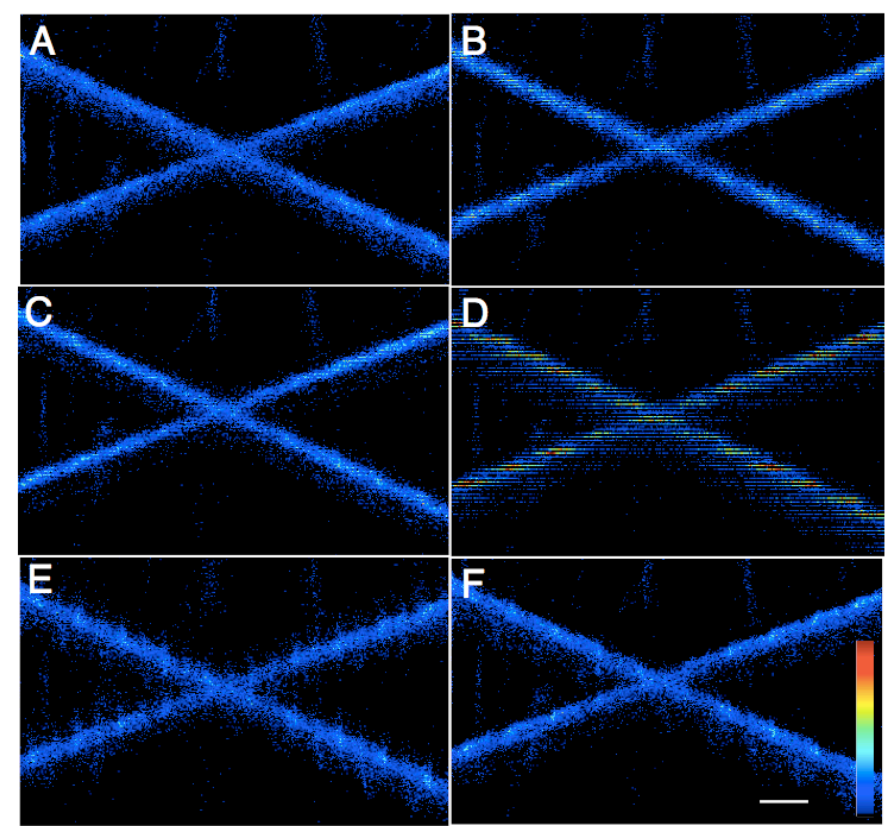

Figure 7. Localization maps using A) Centroiding, B) onset, C) $50 \%$ point, D) peak detection, E) 2D Gaussian fitting and F) 2D fixed Gaussian fitting. Scale bar $1 \mathrm{~mm}$, colour bar 0-12 localizations. Note: horizontal lines noticeable in images are due to effects caused by the original image pixel size.
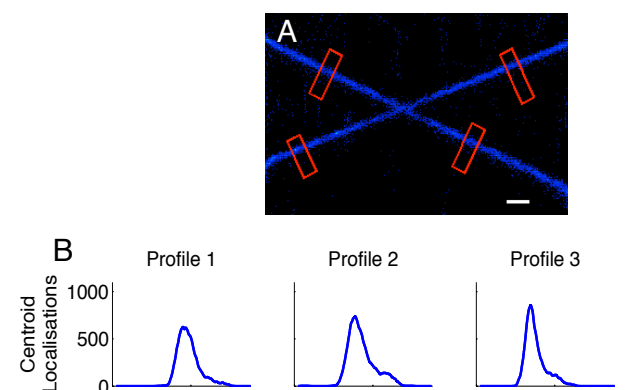

Profile 4
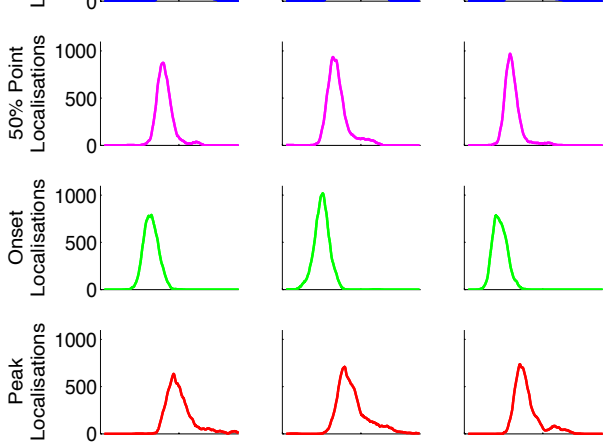

泣
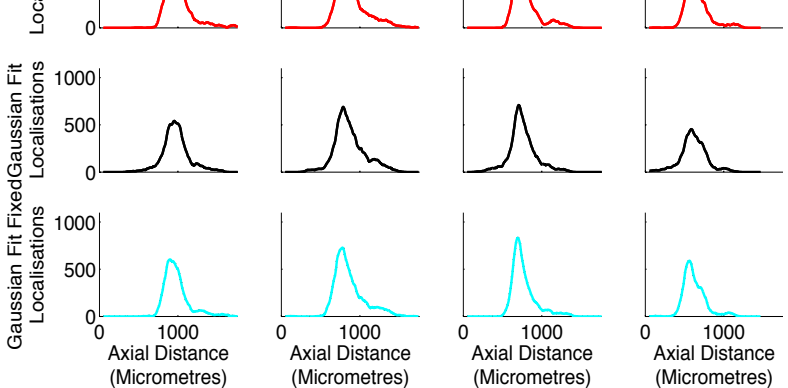

Figure 8. Localization map profiles across the tube diameter from the resulting localization maps. Four $600 \mu \mathrm{m}$ sections across the localization maps, shown in A), are used to generate summed axial profiles for each of the localization methods, shown in B).

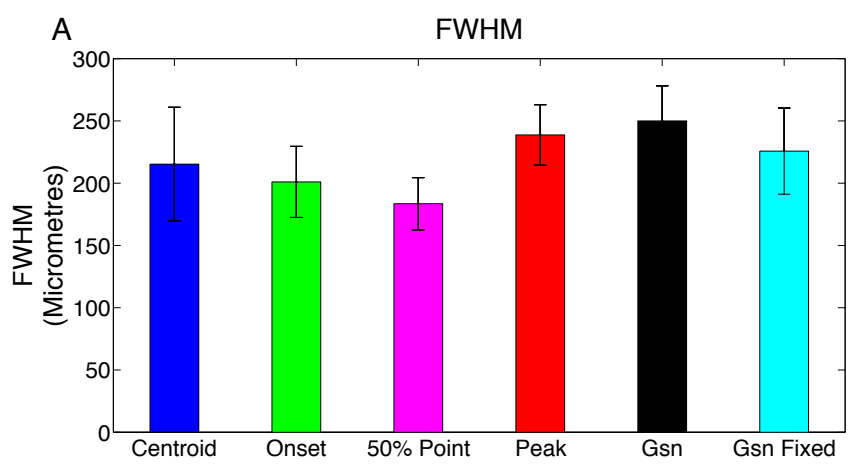

B

IQR
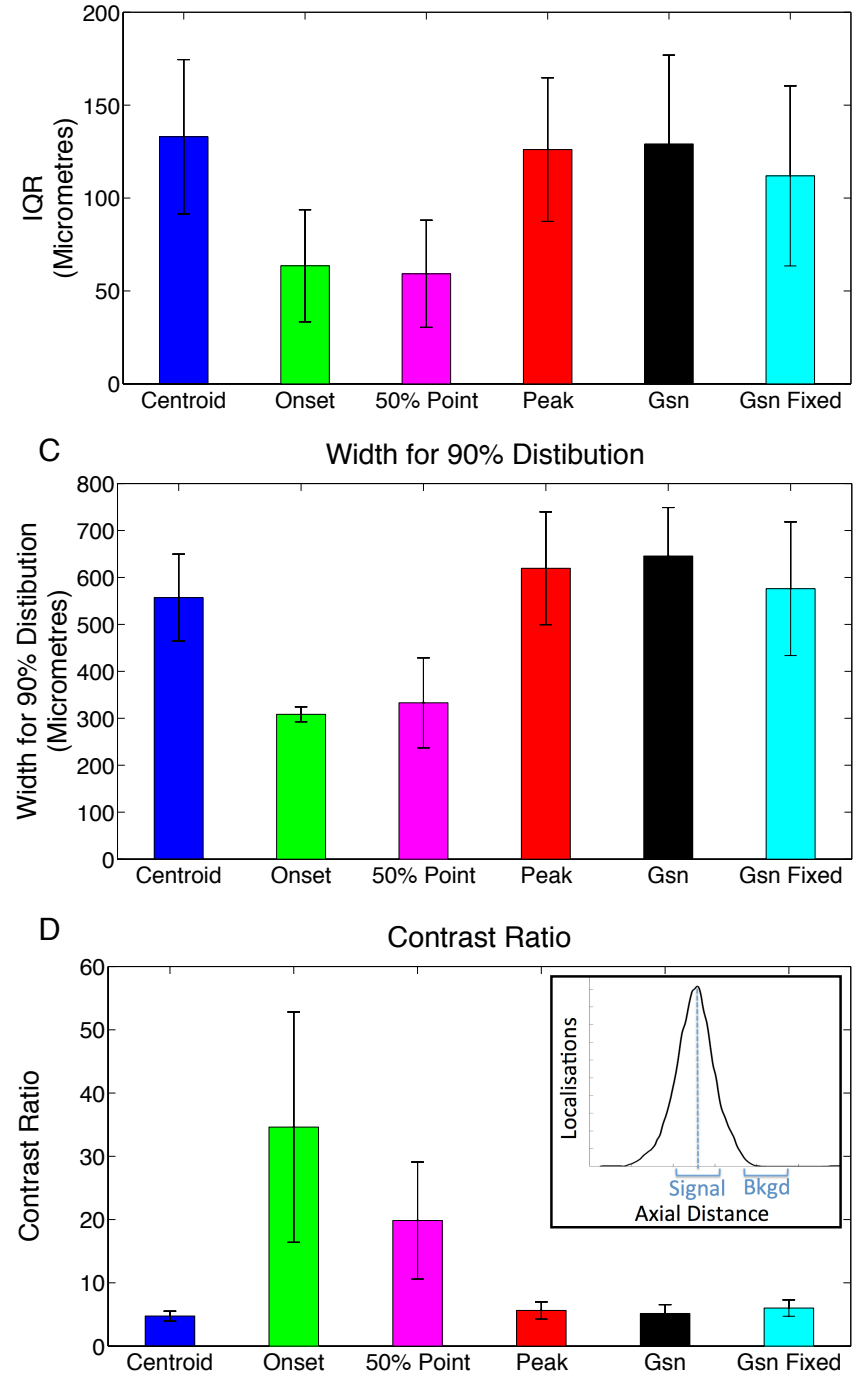

Figure 9. Localization methods applied to bubble signals. The localization distribution within the profile FWHM, and the width for which $90 \%$ of localizations exist provide an indication of the proportion of inaccurate localizations. Calculating the centroid, peak or Gaussian fit of the signal is shown to generate inaccurate localizations outside of the tube diameter, while finding the beginning of the signal by detecting the beginning section of each waveform demonstrates more accurate localizations shown by the spread of localizations remaining largely within the nominal tube diameter $(200 \mu \mathrm{m})$. The contrast ratio demonstrates considerable improvement using onset to all other localization methods. 


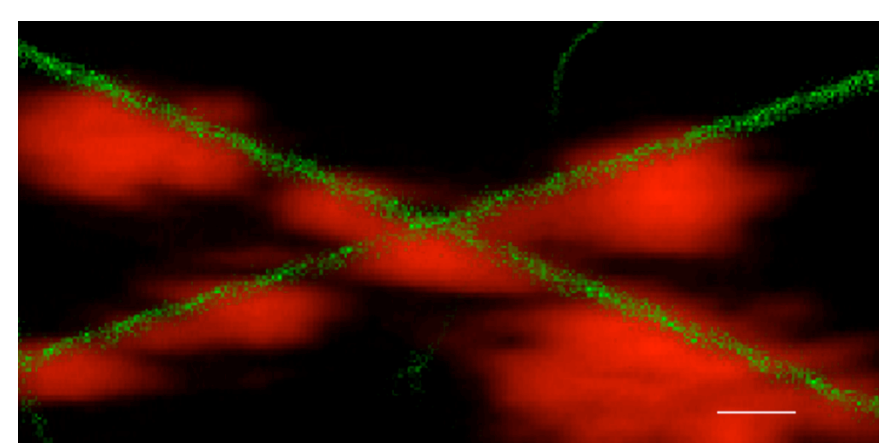

Figure 10. Overlay of the localization map (green) on a diffraction limited image frame with a high microbubble concentration (red). Scale bar $1 \mathrm{~mm}$.

\section{DISCUSSION}

The fundamental accuracy with which a microbubble's position can be determined with any localization method is limited by several noise factors, including Gaussian noise, readout noise, nonlinear artifact, and the effect of pixilation.

Nonetheless, the accuracy of the localization method employed is crucial to the performance of US-SR and has thus been investigated in this study.

Calculating the centroid, Gaussian fit, or peak of the signal is shown experimentally to generate inaccurate localizations far outside the nominal tube diameter, while detecting the onset of the signal by implementing a noise floor threshold or $50 \%$ point demonstrates more accurate localization results. This is demonstrated by a large proportion of the localization distribution remaining within the expected tube diameter, resulting in an improvement in local CR by a factor of at least 5.8 and 3.9 respectively compared to centroiding, peak detection, and Gaussian fitting techniques. This is likely to be due to the these latter methods having a sensitivity to the intensity and duration of the received signal; these are characteristics which vary widely across a single dataset due to the microbubble size distribution and composition, the fluctuations in the transmit pressure field across the field of view, and the microbubble's proximity to nearby objects. Furthermore, peak detection has the disadvantage of retaining sensitivity to noise; this may be improved by the use of a smoothing filter however its dependence on the smoothed signal intensity and shape will remain.

The 50\% point presented a smaller FWHM and IQR measure than that seen using the onset method.
Since these values were extracted from tube-based experiments, these measurements are representative of the spread of localizations over the tube diameter and not necessarily errors in localizations. Localizations which spread beyond the tube diameter, however, represent inaccurate localization positions and are presented in the quantitative measures using the width for $90 \%$ of localizations, and the $\mathrm{CR}$. The $\mathrm{CR}$ value demonstrates the improved accuracy of the onset method over the $50 \%$ point method.

Simulated results similarly suggest that centroiding and variable-width Gaussian fitting are strongly affected by elongated or distorted waveforms, and resonance behavior as shown in Figure $3 \mathrm{C}$ and D, where localizations are biased towards the latter end of the signal; this produces inaccurate microbubble localizations further away from the transducer. Peak detection showed the largest spread of errors in simulations; this method is not only affected by elongated signals, but additionally inaccurate localizations can result when the envelope of the waveform is heavily distorted or in the presence of noise. The $50 \%$ point method reduces the effect of noise and elongated signals compared to the peak detection algorithm; however, it is affected by envelope distortions which can be significant in case of PI for resonant bubbles. The onset method is less dependent on the amplitude and elongation of bubble signals than all the other tested methods, and hence is more representative of the time-of-flight measure from the insonated object. In practice, care must be taken to measure the noise level of the system to ensure localizations generated using this method are robust.

In general, simulated results indicate that, at this pressure level, implementing an inaccurate localization method, such as 2D Gaussian fitting, centroiding or peak detection, can introduce errors in localization in the $0.2-0.4 \mathrm{~mm}$ range when considering the IQR, and in the $\sim 0.9 \mathrm{~mm}$ range when considering the $99.3 \%$ distribution width using 2.3 MHz transmit pulse and PI. This error could however be considerably reduced by using the signal onset method.

While valuable for illustrating the relative 
difference in performance between the different localization methods, the simulations underestimate a number of factors that are present in a realistic setting. These include differences in simulated bubble behavior compared to the in vitro set-up, such as signal damping due to the proximity of nearby objects or boundaries [34],[35] and possible variations in bubble shell composition [36],[37], both of which may reduce the potential signal size which exceeds the noise floor. Furthermore, ideal, compression-only resonance behaviour as predicted by the simulation may not always occur in reality. This means, non-linear microbubble behaviour and hence the signal strength after pulse inversion will be weaker for most of the microbubbles, especially those off-resonance. In addition, the bubble position within the field of view will influence the insonating pressure experienced by the bubble; pressures experienced by bubbles are therefore likely to be lower than that measured at the focus and subsequently used in simulations. In reality, the population of microbubbles which provide a signal strong enough to be detected in the resulting image data and subsequently extracted as single bubble signals is likely to be narrower than the full microbubble range analysed in simulation results. The combination of these factors indicates that the simulation demonstrates a 'worst case scenario', and the spread of localisation errors is likely to be lower for experiments.

Effects of varying transmit pressure have not yet been included in this study and could be investigated in future work. There is likely to be a compromise between increased measurement SNR and potential microbubble destruction, as well as signal elongation. Since US-SR is a method relying upon the accumulation of information from flow in the microvasculature over time, the destruction of microbubbles should be avoided.

The distribution of errors may be reduced by using only a subset of the received signals; however, which subset would provide improved accuracy is yet to be investigated, or possibly by using a mono-dispersed microbubble population. This may provide a more uniform backscatter behavior, and thus reduce the potential variation in localization error.

It is generally acknowledged that increasing the US imaging aperture improves image quality; however, this is only true up to a certain extent. With larger apertures and a fixed target range, the image quality becomes poorer. In many cases, this can be attributed to the effect of sound speed inhomogeneities within different media in the scan plane, called aberration. However, an additional complication can be ascribed to the combination of point scatterer echoes from different angular directions. Images resulting from the combination of signals from active apertures with large angular separation can introduce errors in determining the axial locations of targets from their echoes due to differences in the directionality of backscatter signals. As an example, in the case of a $90^{\circ}$ separation, the compounded image will resemble two partially overlapping perpendicular ellipsoids, both beginning at the point scatterer position. Details of the transmit and receive acquisition parameters should be therefore considered when compounding data for localization procedures.

The use of higher frequency transmission allows for a shorter spatial pulse length and higher axial resolution. It is therefore likely to reduce the potential spread of errors caused by variations in signal responses. Importantly, however, the use of lower transmission frequencies is preferable to demonstrate improvement in resolution at large depths where many high resolution techniques fail.

It is also important to note that interfering or overlapping signals resulting from multiple bubbles within the resolution limit can cause large position errors; interference effects mean that the chosen feature of the received signal cannot accurately be identified, and thus only spatially isolated microbubble signals should be localized for US-SR imaging. Therefore, rejection of these signals was performed prior to localization in this work using an empirically determined upper size threshold. In the future, more sophisticated and automated approaches to defining inclusion/exclusion criteria should be investigated.

In clinical situations, tissue attenuates higher frequencies more than lower frequencies, thus 
effectively stretching the pulse length. This has the potential to generate even higher localization errors when using localization techniques that are affected by the signal duration or position of the maximum amplitude, such as Gaussian fitting, centroiding, and peak detection.

The improvement demonstrated using this proposed method reveals that the start of the signal is more representative of bubble location than other wave features tested, and thus onset detection is crucial for optimisation of super-resolution localisation. Using the onset method in an in vivo setting will require careful determination of the estimated noise level. In future work, we hope to investigate how to best optimise this onset definition in vivo.

This study is based on image data collected from a clinical imaging system. This method of superresolution therefore provides the advantage that it can be performed on patient data directly from the clinic without the need for a research system or additional equipment. The method, however, is of course transferable to experimental studies allowing RF data extraction [26],[27],[44]. In this case, the detection of the signal onset in each RF line should similarly provide the most accurate representation of relative bubble position since the dependence of other localization methods on signal elongation, distortion and noise will be analogous to those shown in this work, and is demonstrated here within simulations.

\section{Conclusion}

Experimental measurements and simulated results suggest that detecting the onset of the backscatter signal from microbubbles, rather than the centroid, peak, Gaussian fit result or 50\% point, will achieve considerably improved accuracy in super-resolution imaging.

\section{REFERENCES}

[1] N. Weidner and J. Folkman, "Tumoral vascularity as a prognostic factor in cancer.," Important Adv. Oncol., pp. 167-90, Jan. 1996.

[2] N. Weidner, J. Folkman, F. Pozza, P. Bevilacqua, E. N. Allred, D. H. Moore, S. Meli, and G. Gasparini, "Tumor angiogenesis: a new significant and independent prognostic indicator in early-stage breast carcinoma.," J. Natl. Cancer Inst., vol. 84, no. 24, pp. 1875-87, Dec. 1992.

[3] American Diabetes Association, "Peripheral Arterial Disease in People with Diabetes," Diabetes Care, vol. 26, no. 12, pp. 3333-3341, 2003.
[4] M. J. Fowler, "Microvascular and Macrovascular Complications of Diabetes," Clin. Diabetes, vol. 26, no. 2, pp. 77-82, Apr. 2008.

[5] P. E. Norman, J. W. Eikelboom, and G. J. Hankey, "Peripheral arterial disease: prognostic significance and prevention of atherothrombotic complications.," Med. J. Aust., vol. 181, no. 3, pp. 150-4, 2004.

[6] D. Cosgrove and N. Lassau, "Imaging of perfusion using ultrasound.," Eur. J. Nucl. Med. Mol. Imaging, vol. 37 Suppl 1, pp. S65-85, Aug. 2010.

[7] H. Becher and P. N. Burns, Handbook of contrast echocardiography: left ventricular function and myocardial perfusion. Springer, 2000.

[8] M. A. Pysz, K. Foygel, C. M. Panje, A. Needles, L. Tian, and J. K. Willmann, "Assessment and monitoring tumor vascularity with contrast-enhanced ultrasound maximum intensity persistence imaging.," Invest. Radiol., vol. 46, no. 3, pp. 187-95, Mar. 2011.

[9] D. Cosgrove, "Ultrasound contrast agents: an overview.," Eur. J. Radiol., vol. 60, no. 3, pp. 324-30, Dec. 2006.

[10] G. R. Lockwood, D. H. Turnball, D. A. Christopher, and F. S. Foster, "Beyond $30 \mathrm{MHz}$ [applications of high-frequency ultrasound imaging]," IEEE Eng. Med. Biol. Mag., vol. 15, no. 6, pp. 60-71, 1996.

[11] S. T. Hess, T. P. K. Girirajan, and M. D. Mason, "Ultra-high resolution imaging by fluorescence photoactivation localization microscopy.," Biophys. J., vol. 91, no. 11, pp. 4258-72, Dec. 2006.

[12] M. G. L. Gustafsson, "Nonlinear structured-illumination microscopy: wide-field fluorescence imaging with theoretically unlimited resolution.," Proc. Natl. Acad. Sci. U. S. A., vol. 102, no. 37, pp. 13081-6, Sep. 2005.

[13] S. W. Hell, "Far-Field Optical Nanoscopy," Science (80-. )., vol. 316, no. $5828,2007$.

[14] T. A. Klar and S. W. Hell, "Subdiffraction resolution in far-field fluorescence microscopy.," Opt. Lett., vol. 24, no. 14, pp. 954-6, Jul. 1999.

[15] E. Betzig, G. H. Patterson, R. Sougrat, O. W. Lindwasser, S. Olenych, J. S. Bonifacino, M. W. Davidson, J. Lippincott-Schwartz, and H. F. Hess, "Imaging intracellular fluorescent proteins at nanometer resolution.," Science, vol. 313, no. 5793, pp. 1642-5, Sep. 2006.

[16] M. J. Rust, M. Bates, and X. Zhuang, "Sub-diffraction-limit imaging by stochastic optical reconstruction microscopy (STORM)," Nat. Methods, vol. 3, no. 10, pp. 793-796, 2006.

[17] J. Enderlein, E. Toprak, and P. R. Selvin, "Polarization effect on position accuracy of fluorophore localization," Opt. Express, vol. 14, no. 18 , p. 8111, Sep. 2006.

[18] R. J. Ober, S. Ram, and E. S. Ward, "Localization accuracy in singlemolecule microscopy.," Biophys. J., vol. 86, no. 2, pp. 1185-200, Feb. 2004.

[19] B. Huang, M. Bates, and X. Zhuang, "Super-resolution fluorescence microscopy.," Annu. Rev. Biochem., vol. 78, pp. 993-1016, Jan. 2009.

[20] O. M. Viessmann, R. J. Eckersley, K. Christensen-Jeffries, M. X. Tang, and C. Dunsby, "Acoustic super-resolution with ultrasound and microbubbles.," Phys. Med. Biol., vol. 58, no. 18, pp. 6447-58, Sep. 2013.

[21] K. Christensen-Jeffries, R. J. Browning, M.-X. Tang, C. Dunsby, and R. J. Eckersley, "In Vivo Acoustic Super-Resolution and SuperResolved Velocity Mapping Using Microbubbles," IEEE Trans. Med. Imaging, 2015.

[22] M. Siepmann, G. Schmitz, J. Bzyl, M. Palmowski, and F. Kiessling, "Imaging tumor vascularity by tracing single microbubbles," in 2011 IEEE International Ultrasonics Symposium, 2011, pp. 1906-1909.

[23] C. Errico, J. Pierre, S. Pezet, Y. Desailly, Z. Lenkei, O. Couture, and M. Tanter, "Ultrafast ultrasound localization microscopy for deep super-resolution vascular imaging," Nature, vol. 527, no. 7579, pp. 499-502, Nov. 2015.

[24] D. Ackermann, G. Schmitz, and S. Member, "Detection and Tracking of Multiple Microbubbles in Ultrasound B-Mode Images," vol. 63, no. 1, pp. 72-82, 2016.

[25] F. Lin, S. E. Shelton, D. Espíndola, J. D. Rojas, G. Pinton, and P. A. Dayton, "3-D Ultrasound Localization Microscopy for Identifying Microvascular Morphology Features of Tumor Angiogenesis at a Resolution Beyond the Diffraction Limit of Conventional Ultrasound," Theranostics, vol. 7, no. 1, pp. 196-204, 2017.

[26] Y. Desailly, O. Couture, M. Fink, and M. Tanter, "Sono-activated ultrasound localization microscopy," Appl. Phys. Lett., vol. 103, no. 17, p. 174107, 2013.

[27] M. a O’Reilly and K. Hynynen, "A super-resolution ultrasound method 
for brain vascular mapping.," Med. Phys., vol. 40, no. 11, p. 110701, Nov. 2013.

[28] Y. Desailly, J. Pierre, O. Couture, and M. Tanter, "Resolution limits of ultrafast ultrasound localization microscopy.," Phys. Med. Biol., vol. 60, no. 22, pp. 8723-40, Nov. 2015.

[29] S. Hilgenfeldt, D. Lohse, and M. Zomack, "Response of bubbles to diagnostic ultrasound: a unifying theoretical approach," Eur. Phys. J. B, vol. 4, no. 2, pp. 247-255, Aug. 1998.

[30] Y. Gong, M. Cabodi, and T. Porter, "Size-dependent backscatter coefficient from lipid-coated monodisperse microbubbles," J. Acoust. Soc. Am., vol. 131, no. 4, pp. 3324-3324, Apr. 2012.

[31] J. M. Gorce, M. Arditi, and M. Schneider, "Influence of bubble size distribution on the echogenicity of ultrasound contrast agents: a study of SonoVue.," Invest. Radiol., vol. 35, no. 11, pp. 661-71, Nov. 2000.

[32] S. M. van der Meer, M. Versluis, D. Lohse, C. T. Chin, A. Bouakaz, and N. d. Jong, "The resonance frequency of SonoVue as observed by high-speed optical imaging," in IEEE Ultrasonics Symposium, 2004, 2004, vol. 1, pp. 343-345.

[33] V. Sboros, C. A. MacDonald, S. D. Pye, C. M. Moran, J. Gomatam, and W. N. McDicken, "The dependence of ultrasound contrast agents backscatter on acoustic pressure: theory versus experiment," Ultrasonics, vol. 40, no. 1, pp. 579-583, 2002.

[34] V. Garbin, D. Cojoc, E. Ferrari, E. Di Fabrizio, M. L. J. Overvelde, S. M. van der Meer, N. de Jong, D. Lohse, and M. Versluis, "Changes in microbubble dynamics near a boundary revealed by combined optical micromanipulation and high-speed imaging," Appl. Phys. Lett., vol. 90, no. 11, p. 114103, Mar. 2007.

[35] H. J. Vos, B. Dollet, J. G. Bosch, M. Versluis, and N. de Jong, "Nonspherical vibrations of microbubbles in contact with a wall: a pilot study at low mechanical index.," Ulsnd Med. Biol., vol. 34, no. 4, pp. 685-8, Apr. 2008.

[36] S. Dicker, M. Mleczko, M. Siepmann, N. Wallace, Y. Sunny, C. R. Bawiec, G. Schmitz, P. Lewin, and S. P. Wrenn, "Influence of Shell Composition on the Resonance Frequency of Microbubble Contrast Agents," Ultrasound Med. Biol., vol. 39, no. 7, pp. 1292-1302, 2013.

[37] H. Leong-Poi, J. Song, S.-J. Rim, J. Christiansen, S. Kaul, and J. R.
Lindner, "Influence of microbubble shell properties on ultrasound signal: Implications for low-power perfusion imaging," J. Am. Soc. Echocardiogr., vol. 15, no. 10, pp. 1269-1276, 2002.

[38] N. de Jong, A. Bouakaz, and P. Frinking, "Basic Acoustic Properties of Microbubbles," Echocardiography, vol. 19, no. 3, pp. 229-240, Apr. 2002.

[39] S. Qin and K. W. Ferrara, "The natural frequency of nonlinear oscillation of ultrasound contrast agents in microvessels.," Ultrasound Med. Biol., vol. 33, no. 7, pp. 1140-8, Jul. 2007.

[40] V. Sboros, "A review of Single Microbubble Acoustics," no. August, pp. $1-5,2010$.

[41] J. Tu, J. Guan, Y. Qiu, and T. J. Matula, "Estimating the shell parameters of SonoVue ${ }^{\circledR}$ microbubbles using light scattering," $J$. Acoust. Soc. Am., vol. 126, no. 6, p. 2954, 2009.

[42] P. Marmottant, S. van der Meer, M. Emmer, M. Versluis, N. de Jong, S. Hilgenfeldt, and D. Lohse, "A model for large amplitude oscillations of coated bubbles accounting for buckling and rupture," J. Acoust. Soc. Am., vol. 118, no. 6, p. 3499, 2005.

[43] S. M. Van Der Meer, M. Versluis, D. Lohse, C. T. Chin, A. Bouakaz, and N. De Jong, "The resonance frequency of SonoVue ${ }^{\mathrm{TM}}$ as observed by high-speed optical imaging," 2004 IEEE Ultrason. Symp., vol. 0, no. c, pp. 343-345, 2004.

[44] K. Christensen-Jeffries, J. Brown, p. Aljabar, M.-X. Tang, C. Dunsby, and R. J. Eckersley, "3-D In Vitro Acoustic Super-Resolution and Super-Resolved Velocity Mapping Using Microbubbles," IEEE Trans. Ultr. Ferr. Freq. Contr., 2017.

The data supporting this research are openly available from the King's College London research data archive, doi:10.18742/RDM01-224. Recommended citation: K. M. Christensen-Jeffries, J. Brown, P. Aljabar, R. Eckersley, S. Harput, P. Wells, C. Dunsby, M. Tang (2017). Contrast enhanced ultrasound in vitro data. King's College London. Dataset. http://doi.org/doi:10.18742/RDM01-224. 